\title{
MMAPS: Missing-Mass A-Prime Search
}

\author{
Jim Alexander \\ ${ }^{1}$ Cornell University
}

\begin{abstract}
An experiment is proposed to search for dark photons via electron-positron annihilation into photon plus dark photon. The dark photon would be reconstructed in a missing-mass technique, thus ensuring complete model independent. The Cornell University synchrotron can provide a positron beam suitable to probe the interesting mass range for dark photons. A detector based on CsI calorimetry is proposed, and the potential experimental reach in coupling constant and dark photon mass is shown.
\end{abstract}

\section{Introduction and Motivation}

Although dark matter is generally thought to be noninteracting, a feeble analog of the standard model electromagnetic interaction could be active in the dark sector, and could be produced in $e^{+} e^{-}$annihilations. Many experiments are currently in progress or under construction to search for the "dark photon". The experiment discussed here, based on the $5.3 \mathrm{GeV}$ positron beam at Cornell University's Wilson Laboratory (soon to be upgraded to $6.0 \mathrm{GeV}$ ), would extend the current limits in dark photon parameter space by almost two orders of magnitude. The experiment employs a missing-mass strategy [2] to search for $e^{+} e^{-} \rightarrow \gamma A^{\prime}$, reconstructing the dark photon mass with a missing mass technique that depends only on the $e^{+}, e^{-}$, and $\gamma$ kinematics. No assumptions about the $A^{\prime}$ decay mode need be made. A detector has been proposed, constructed primarily from components recovered from the retired CLEO and Babar experiments; similar detectors proposed at LNF (PADME) [3] and the Budker Institute (VEPP3) [4] could also serve. The initially proposed experiment will be referred to here as the "Missing-Mass A-Prime Search", or MMAPS.

\section{Experimental Strategy}

In the MMAPS experiment proposed here, we search for the process $e^{+} e^{-} \rightarrow \gamma A^{\prime}$ in events produced by a positron beam incident on a fixed target. The ordinary photon in the final state is observed and its four-momentum measured; with a sufficiently large dataset the unseen $A^{\prime}$ will appear as a bump in the missing-mass spectrum, $M_{\text {miss }}^{2} \equiv\left(p_{e^{+}}+p_{e^{-}}-p_{\gamma}\right)^{2}$.

The figure of merit for a bump-hunting experiment is the sensitivity $S / \sqrt{B}$ at any bump mass $M_{\text {miss }}$. For luminosity $\mathcal{L}$, runtime $T$, and mass resolution $\sigma_{M}$, the figure of merit for an apparatus of acceptance $\mathcal{A}$ can be defined as F.O.M. $\equiv \sqrt{\mathcal{L} T \mathcal{A}} / \sigma_{M \text { rec }}$. The experimental goals are therefore, in broad-brush terms, to obtain the highest possible beam intensity, highest practical acceptance, and the lowest possible mass resolution. 

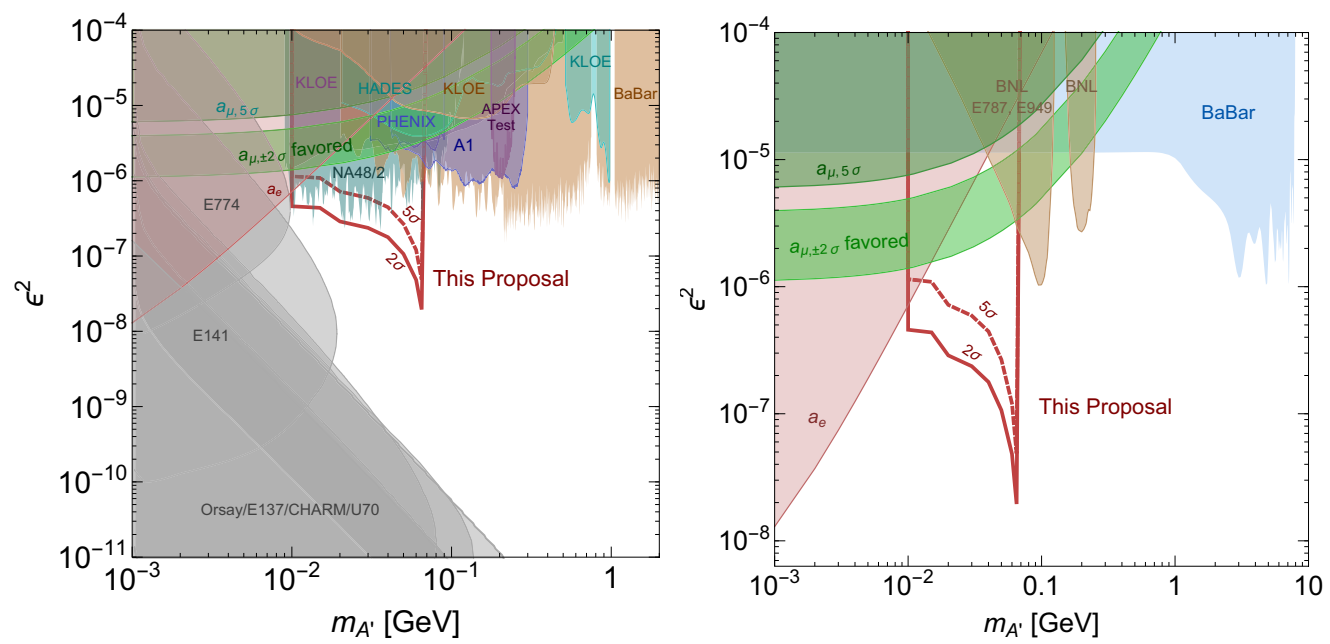

Figure 1. Current experimental bounds[5] on the dark photon mass $M_{A^{\prime}}$ and the square of the coupling constant to electrons and positrons, $\varepsilon^{2}$. Left panel: experiments sensitive to the visible $A^{\prime} \rightarrow e^{+} e^{-}$final state. Right panel: experiments sensitive to invisible $A^{\prime}$ decays. Estimated 95\% c.l. exclusion reach of the experiment proposed here is shown by the teal curve labelled "This Proposal".

MMAPS can be realized at Cornell's Wilson Lab where the existing synchrotron provides a positron source with properties well-matched to the goals of this experiment. A comparable positron beam is not available elsewhere in the world. The positrons will be extracted slowly from the synchrotron over an energy range around $6 \mathrm{GeV}$, and directed to a fixed target; the main detector element, a calorimeter of CsI crystals, is positioned $10 \mathrm{~m}$ further downstream. Figures 2 and 5 illustrate the planned location and configuration of the detector. A small number of magnets need to be designed and fabricated to extract the positron beam from the synchrotron, and some vacuum work is needed when we reposition the existing synchrotron magnets to accomodate new elements.

Most of the detector components will be adapted from existing equipment inherited from previous experiments. The CsI crystals are taken from the now-retired CLEO experiment, and many of the accelerator components already exist and require only reconfiguration or other limited modifications. For improved performance, the photodiodes used to readout the CLEO calorimeter will be replaced by phototubes.

The synchrotron today fills the CESR storage ring with $e^{+}$and $e^{-}$for x-ray production, and the experiment proposed here will operate entirely in the shadow of the x-ray program, providing positrons for dark photons only in the 3-minute intervals between positron top-ups carried out for the x-ray program. A modest duty factor of $75 \%$ is expected to cover the edge-effects associated with switching between two kinds of synchrotron operation.

The anticipated performance of MMAPS is based on GEANT4 simulation [6]. The sensitivity is shown in Fig. 1, which indicates (in brown) the region of the parameter space of coupling constant versus mass that this experiment can exclude at $2 \sigma$ or $5 \sigma$ with a run of $10^{7}$ seconds, together with the existing set of exclusion limits from other experiments. 


\section{Beam Extraction}

To provide the positron beam needed for the MMAPS project we use the Cornell synchrotron [7]. For MMAPS we will extract positrons from the synchrotron and deliver them to a fixed target; the acceleration and extraction take place in the shadow of xray operations, ensuring efficient use of the machine without interference in xray production.

Positrons are created and brought to $150 \mathrm{MeV}$ in a short linac prior to injection into the synchrotron, where they are accelerated to $6 \mathrm{GeV}$. The average positron current in the synchrotron is $I_{\text {avg }}^{+} \sim 7.7 \mathrm{nA}$. The synchrotron acceleration cycle repeats 60 times per second; the high energy beam is normally extracted at the peak of the ramp. The orbital period is $2.52 \mu$ s, with 180 bunches at $14 \mathrm{~ns}$ intervals. Maximum positron current is realized in 15 bunches spaced $168 \mathrm{~ns}$ apart. A bunch completes $\sim 3000$ turns from injection to extraction at the peak of cycle.

For routine injection into the CESR storage ring, the entire beam in the synchrotron is extracted on a single turn by a fast kicker. For MMAPS operation a slow spill is prefered to minimize pileup; we plan to use a resonant extraction scheme, whereby the synchrotron beam is slowly extracted over about 1000 turns $(\sim 2.5 \mathrm{~ms})$. To accomplish this, quadrupoles shift the betatron tune of the synchrotron beam onto a third-integer $\left(Q=10 \frac{2}{3}\right)$, and a localized sextupole field drives a nonlinear resonance that shrinks the stable region of phase space in a controlled manner. The amplitude of the particles outside of the shrinking stable region increases over the course of several turns, until the horizontal displacement of particles outside the stable region is sufficient to clear a thin septum. The displacement is increased by the transverse kick from the first thin septum. Two stronger septa then steer the particles into the extraction beam line. A resonantly extracted electron beam was used in fixed target experiments at Cornell in the period 1968-1979 [8], and is also in routine use today at the $3.5 \mathrm{GeV}$ ELSA ring in Bonn [9].



Figure 2. Approximate configuration of dark photon apparatus. Plan view shows south arc of synchrotron and CESR storage ring with MMAPS detector and beamline superimposed.

Extraction efficiency depends on our ability to manipulate the betatron tune of the synchrotron with quadrupoles, the strength of the resonance with the sextupole, and the performance of the extraction septa. Simulation studies indicate that the extraction efficiency will be around $80 \%$ with an 
$0.8 \mathrm{~mm}$ thick septum, which is consistent with performance achieved under a similar extraction strategy in the pre-1979 era. For a fixed extraction efficiency, the emittance of the extracted beam scales with septum thickness; the simulations show that the extracted beam emittance is well below the experimental requirement, even with a $1 \mathrm{~mm}$ septum. To minimize the emittance of the extracted beam, the high energy beam is moved to within $\sim 10 \mathrm{~mm}$ of the septum with a 3-component pulsed bump that turns on at the start of the extraction cycle. Evolution of the phase space of the positron beam through acceleration cycle is shown in Fig. 3.

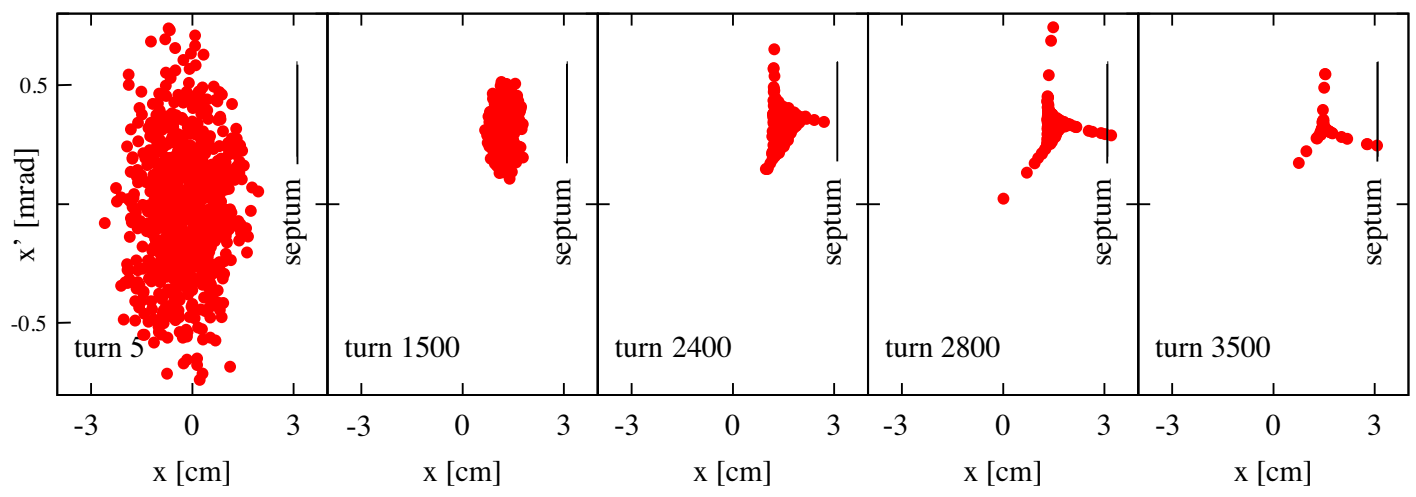

Figure 3. Beam phase space in the synchrotron at five points during the acceleration and extraction cycle. At turn 5 (leftmost panel) injection from the linac into the sychrotron the phase space fills the aperture. By turn 1500 the emittance has shrunk as the particles gained energy, and the bump has shifted it towards the extraction septum. Around turn 2400, the pulsed quadrupole and sextupole shrink the stable phase space. During turns 2500-3500, (right two panels) the particles outside the stable region step beyond the septum and into the extraction line.

The capability to resonantly extract beam from the synchrotron requires installation of a new sextupole and two quadrupole magnets, together with associated current sources. A single sextupole will drive the resonance. One trim quadrupole will be needed to shift the horizontal betatron tune onto the resonance and another to fix the vertical tune. To move the beam close to the first septum for extraction, a closed-orbit bump is generated with the existing trim windings on three of the synchrotron magnets. The uniformity with which positrons are extracted through the $\sim 1000$ turn extraction cycle is sensitive to the tune shift (quadrupole), sextupole strength, and bump amplitude from one turn to the next. Programmable power supplies for the extraction magnets will enable the requisite fine tuning.

The first septum is relatively thin. It's kick is enough to separate the outgoing particles from the circulating beam so that they clear a second, thicker septum, which then provides sufficient kick to direct the beam into the extraction line. The septum magnets will be inserted between synchrotron magnets as shown in Figure 2.

The sextupole, quadrupoles, and trim windings for the closed-orbit bump will all require programmable current sources. Uniform extraction over a thousand turns is sensitive to the proximity to the third integer resonance and the strength of the sextupole. The septa on the other hand, can be powered in series with the synchrotron dipoles, in the same way that the septa are currently powered to extract beam for injection into CESR. The beam energy varies over the course of the extraction cycle, but at any moment the synchrotron dipole magnetic field is precisely known, given the phase in the cycle, and the beam energy is fixed by the synchrotron $\sim 1 \%$ energy acceptance. A measurement of the synchrotron beam position, with the turn by turn capable beam position monitor, gives the beam 
energy to $<0.1 \%$. The magnets in the extraction beam line will likewise be powered in series with the synchrotron magnets in order to maintain energy independent focusing and steering of the extracted beam.

The beam that exits the synchrotron will see a pair of dipole correctors and quadrupoles in the extraction beam line to steer and focus it onto the target. The quadrupoles will be assembled from synchrotron laminations. Combined function magnets and quadrupoles will be powered in series with the synchrotron magnets so that the energy of the beam line is consistent with the beam energy. The dipole correctors will be air core (low impedance) magnets that permit near turn by turn correction of the varying launch angle of the extracted particles.

A model of the synchrotron guide field [10], including the magnets required for resonant extraction, was used to study resonant extraction. The time-dependent fields of the sextupole, quadrupole, and displacment bump magnets were tuned in the simulation to produce a nearly uniform spill, thus defining the required time dependence of the magnets.

To benchmark the simulation results with measurements, we have recently installed a high bandwidth stripline detector in the synchrotron to measure turn-by-turn transverse position of the accelerating beam. The measured displacement corresponds to the turn dependent energy offset. We are preparing to install two additional strip line detectors near the extraction sextupole and septa respectively to measure phase advance.

The synchrotron will continue to serve as the injector for the storage ring for its primary role as an xray source. Every three minutes the delivery of positrons to the MMAPS target will be interrupted for top-off of the positron beam in the storage ring; this will result in a duty cycle for MMAPS operations of $80 \%$. We also anticipate that with a conservative design of the components for the resonant extraction scheme, that we will extract $\sim 30 \%$ of the positrons produced in the linac, corresponding to an average current $\left\langle I_{+}\right\rangle \sim 2.3 \mathrm{nA}$.

\subsection{The External Beam}

A measurement of the missing mass, $M_{\text {miss }}^{2} \equiv\left(p_{e^{+}}+p_{e^{-}}-p_{\gamma}\right)^{2}$, requires knowledge of both the final and initial state kinematics. The extraction system described above will provide an incoming positron beam whose energy, direction, and position lie well within the specifications, $\sigma_{E_{+}}<15 \mathrm{MeV}$, $\theta_{+} \ll 0.4 \mathrm{mrad}$, and $\sigma_{x}, \sigma_{y} \ll 4 \mathrm{~mm}$. For beam parameters within these limits, the spread in beam phase space has no discernible impact on missing mass resolution.

The beam extracted from the synchrotron needs to be steered to the target. The fast BPMs will be used for tuning the beam extraction and monitoring the pass through the exit beam line with a $160 \mathrm{~ns}$ response time. We plan to use four beam monitors in the vacuum channel between the accelerator and production target and an additional one in front of the beam dump. Each monitor will be of the "SciFi" type [11] will have 64 scintillator fibers with multianode PMT readout.[12]

The $2.5 \mathrm{msec}$ beam spill repeats every $\frac{1}{60} \mathrm{sec}=16.7 \mathrm{msec}$, and each spill is further substructured into 15,000 bunches, uniformly spaced by $168 \mathrm{~ns}$; each bunch delivers about 17,000 positrons to the target. Given a Beryllium target of $12.7 \mathrm{~mm}$ length, the luminosity can be calculated. We distinguish between the average luminosity, which determines final statistical sensitivity for the experiment, from the peak luminosity, which drives the occupancy in the calorimeter during the spill:

$$
\mathcal{L}_{\mathrm{av}}=0.9 \times 10^{34} \mathrm{~cm}^{-2} \mathrm{~s}^{-1} \quad \mathcal{L}_{\mathrm{pk}}=6.1 \times 10^{34} \mathrm{~cm}^{-2} \mathrm{~s}^{-1} .
$$

\section{Detector}

The extracted beam line directs the positrons onto a beryllium target, and a calorimeter sitting $10 \mathrm{~m}$ further downstream measures the energy and direction of photons produced by the $e^{+} e^{-}$collisions. 


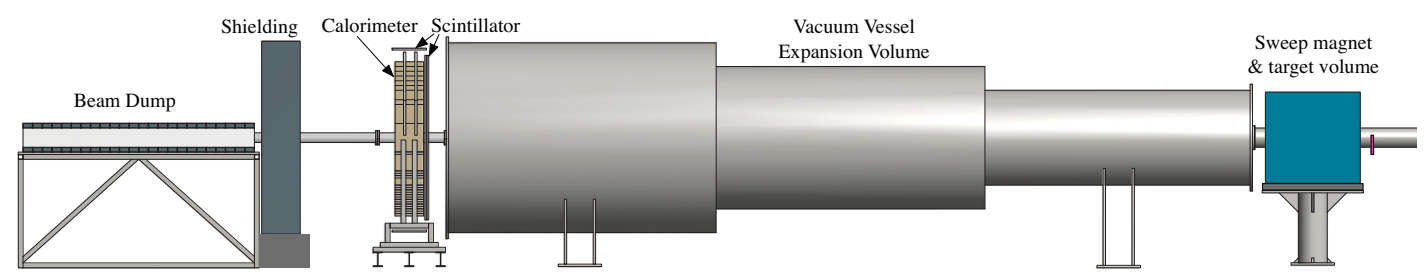

Figure 4. Elevation view of MMAPS.

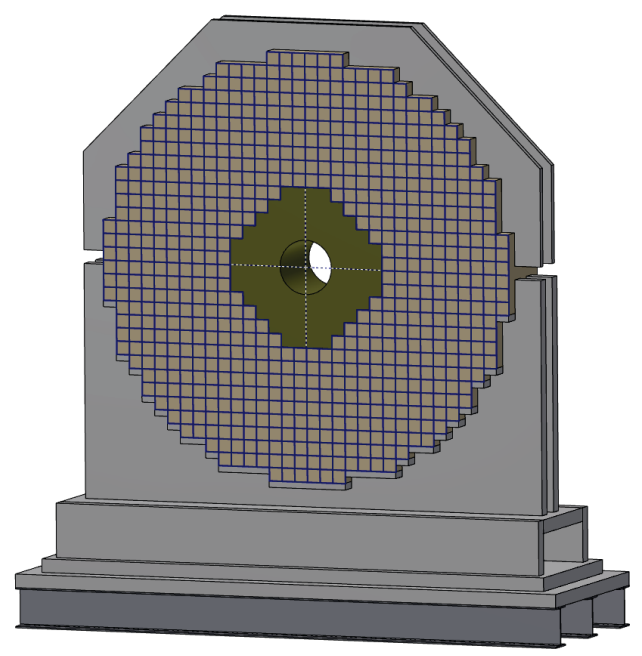

Figure 5. Front view of crystal calorimeter.

The experimental setup, illustrated in Fig. 5, includes a large vacuum vessel between the target and calorimeter, a scintillator veto wall to exclude charged particles, and a beam dump. The active area of the calorimeter and veto wall covers a solid angle of $2^{\circ}-5^{\circ}$ in the lab frame (roughly $136^{\circ}-161^{\circ}$ in the CM frame).

\subsection{Calorimeter}

The calorimeter is a stack of CsI crystals located $10 \mathrm{~m}$ from the target. The crystals, recovered from the CLEO endcap calorimeters, are $5 \times 5 \times 30 \mathrm{~cm}^{3}$ rectangular solids, well suited to stacking in simple arrays. The inner and outer radii of the annular configuration correspond to lab angles for the photon of $2^{\circ} \lesssim \theta_{\gamma} \lesssim 5^{\circ}$.

The Thallium-doped CLEO CsI crystals have a high scintillation yield of 54,000 photons per MeV. The emission spectrum peaks around $560 \mathrm{~nm}$, with a rise time of $\sim 30 \mathrm{~ns}$ and fall time of $\tau \approx 1 \mu \mathrm{s}$ [13].

The particle identification system in the Babar [14] experiment depended on fast, high-gain, lownoise PMTs [15]. For the emission spectrum of CsI(Tl), the Babar tubes have a quantum efficiency of $8 \%$ which, together with the photon collection efficiency of the crystal+tube system, leads to a photoelectron yield of 600 p.e. per MeV. A typical $10 \mathrm{MeV}$ deposition in one crystal will therefore be measured with $1 \%$ precision (i.e. limited by photoelectron statistics), yielding an order of magnitude 


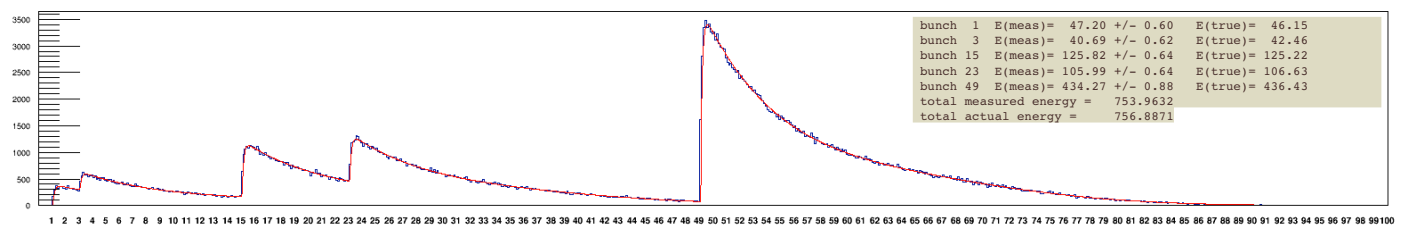

Figure 6. Pulse train after digitization, for 100 beam bunches $(16 \mu \mathrm{s})$. Vertical axis is in photoelectrons per $20 \mathrm{~ns}$ bin; successive beam bunches are separated by 8 bins.

improvement over photodiode performance. Furthermore, the fast response of the PMT gaurantees that it tracks the $\mathrm{CsI}(\mathrm{Tl})$ signal rise time without distortion, a feature that plays a crucial role in handling signal pileup.

Photon energy resolution is the dominant contributor to the missing-mass resolution over most of the angular acceptance. The low intrinsic noise of PMTs ensures that electronic noise (a significant consideration for low energy photons in CLEO) is a minor contribution in MMAPS. In the 5-700MeV range of interest, shower leakage out the back of the crystal is also not important. Thus side-leakage, due the lateral spread of the shower and the consequent fluctuation in energy recovered by clustering, dominates photon energy resolution.

An additional contribution comes from signal pileup, which can affect the measurement of energy deposited in a single crystal when two or more photons happen to deposit energy in the same crystal in nearby beam bunches. The PMT outputs will be continuously digitized by FADCs, and the resulting waveforms analyzed to extract individual photon energies and arrival times. Fig. 6 shows a sample waveform from 16 microseconds of simulated data, showing 5 pulses in one crystal. (The waveform shown is chosen to emphasize extreme pileup conditions: for nominal operating conditions, $\lambda=2.2$, a random sampling of $16 \mu$ s of data has less than $1 \%$ chance of yielding 5 or more pulses.) Decomposing such a pulse train into individual hits is done by an algorithm that takes advantage of the fixed pulse shape and discrete arrival times (coincident with beam bunches).

The average amount of photon pileup in the calorimeter is determined by the peak luminosity discussed in Sec. 3.1, the $1 \mu$ s characteristic time profile of CsI(Tl) emission noted above, and the various background cross sections, acceptances, and spatial distributions. For the intended running conditions, i.e. the peak luminosity as given in Eq. 1, we find the average number of photons in the calorimeter for any beam bunch is $\lambda=2.2$.

\subsection{Calibration}

Calibration constants that convert ADC counts to $\mathrm{MeV}$ must be measured precisely and frequently. Several independent methods allow for plenty of cross-checking:

Two-gamma calibration. In $e^{+} e^{-} \rightarrow \gamma \gamma$ events, only one photon is in the calorimeter acceptance, and its angle $\theta_{\gamma}$ is correlated with its energy: $E_{\gamma}=m_{e} /\left(1-\beta_{C M} \cos \theta_{\gamma}\right)$. The calorimeter geometry will be known to arbitrary precision, allowing us to use the measured angle to determine energy with a resolution $\sigma_{E}=|d E / d \theta| \sigma_{\theta}$. In practice $\sigma_{\theta} \approx 1 \mathrm{mrad}$, and $|d E / d \theta|$ varies from $3 \mathrm{MeV} / \mathrm{mrad}$ at the outer edge to $36 \mathrm{MeV} / \mathrm{mrad}$ at the inner radius of the calorimeter. This process calibrates the entire calorimeter continuously, to a precision well below the intrinsic resolution single-photon resolution: a few seconds of data is sufficient to calibrate every crystal to a fraction of a percent accuracy at the energy corresponding to the crystal's position. 
Cosmic calibration. Each crystal in the calorimeter sees a $\sim 2 \mathrm{~Hz}$ rate of cosmic rays. The most probable value of the energy deposition for a $5 \mathrm{~cm}$ path length in CsI is $28 \mathrm{MeV}$. The actual path length will vary but can be accurately determined event-by-event by using the full crystal array and the two horizontal scintillator layers for tracking. With one day of cosmic tracks, the peak of the Landau distribution can be determined in every crystal to better than $0.5 \%$. This provides a calibration at a fixed low energy point.

Beam calibration. The sweeping magnet plays two roles, the main one being to sweep Bhabhas out of the calorimeter acceptance. The second is to steer low-current, low-energy beam, provided during dedicated calibration runs, at selected crystals. Independently controlled horizontal and vertical coils allow one to choose the $(\phi, \theta)$ direction to strike a selected crystal. This provides absolute calibration across the middle range of energy; but the process is slow and will be done infrequently.

\section{Conclusions and Outlook}

The thinking about dark matter has grown beyond the traditional WIMP paradigm in recent years, opening new vistas and new opportunities for experimental work and, hopefully, discovery. Dark photons in the Mev-to-GeV mass range are a target of particular interest, and many experiments underway or in construction are designed to search for evidence of dark photons. MMAPS is one possible realization of such an experiment, exploiting the missing-mass technique, and depending on the presence of a high-energy, high-intensity positron beam. A beam of this description could be delivered at Cornell University, by equipping the existing synchrotron with additional magnets to create a resonant extraction scheme. This beam could support either MMAPS or one of the similar experiments, such as PADME, that are already in design and construction.

\section{References}

[1] S. Ritz et al., "Building for Discovery: Strategic Plan for U.S. Particle Physics in the Global Context", Report of the Particle Physics Project Prioritization Panel (P5), available online (June 2014).

[2] B. Wojtsekhowski, "Searching for a U-boson with a positron beam", arXiv:0906.5265

[3] Mauro Raggi, Venelin Kozhuharov, P. Valente, "The PADME experiment at LNF", arXiv: 1501.01867

[4] B. Wojtsekhowski, D. Nikolenko, I. Rachek, "Searching for a new force at VEPP-3", arXiv: 1207.5089

[5] Plots courtesy of Rouven Essig; private communication.

[6] S. Agostinelli et al. [GEANT4 Collaboration], "GEANT4: A Simulation toolkit," Nucl. Instrum. Meth. A 506, 250 (2003).

[7] R. R. Wilson, "The 10-GeV TO 20-GeV Cornell Electron Synchrotron,” CS-33, NP-17179.

[8] J. DeWire, H. Edwards, R. Littauer, G. Rouse and R. Sundelin, "An External Beam from the Cornell 10 GeV Synchrotron,” Proceedings 1971 Particle Accelerator Conference, p. 964-965.

[9] W. Hillert, "The Bonn electron stretcher accelerator ELSA: Past and future, Eur. Phys. J. A 28S1, 139 (2006).

[10] D. Sagan, Nucl. Instrum. Methods Phys. Res. A558, 356 (2006). BMAD

[11] "A $1 \mathrm{~mm}$ Scintillating Fibre Tracker Readout by a Multi-anode Photomultiplier" arXiv:1106.5649v1 [physics.ins-det] 28 Jun 2011

[12] http://www.hamamatsu.com/resources/pdf/etd/H8804_TPMH1333E.pdf 
[13] J.D. Valentine, W.W. Moses, S.E. Derenzo, D. Wehe, G. F. Knoll, “Temperature dependence of CsI(Tl) gamma-ray scintillation decay time constants and emission spectrum" NIM A325 (1993) 147 ScienceDirect link

[14] I. Adam et al. [BaBar DIRC Collaboration], "The DIRC particle identification system for the BaBar experiment,” Nucl. Instrum. Meth. A 538, 281 (2005).

[15] EMI 9125FLB17, made by Electron Tube Ltd. 